\title{
A Case of Aspergillosis of the Maxillary Sinus
}

\author{
by \\ Tomonori KAWANA, Hirotsugu YAMAMOTO* and \\ Hirotsugu IZUMI
}

(Received 14 September and accepted 19 October 1987)

Key words: aspergillosis, maxillary sinus, odontogenic infection

\begin{abstract}
A case of aspergillosis of the maxillary sinus followed by odontogenic sinusitis is reported. The Japanese literature on paranasal fungus infection was reviewed and discussed. In Japan, aspergillosis is the most frequently occurring of all paranasal fungus infections, but paranasal aspergillosis followed by odontogenic sinusitis is relatively rare. Most cases of odontogenic paranasal aspergillosis are of the non-invasive type.
\end{abstract}

\section{Introduction}

Fungus infection of the paranasal sinus is relatively rare. In recent years, cases of fungus infection have increased because of the rise in opportunistic infections associated with chemotherapy. Most cases of infection have been treated and reported mainly in the field of otolaryngology. In the field of oral surgery too, odontogenic maxillary sinusitis is often recognized. However, fungus infection caused by odontogenic sinusitis seems to be rare.

We recently encountered a case of Aspergillus infection of the maxillary sinus in a 47-year-old woman, which was followed by odontogenic sinusitis. The literature of the disease is reviewed and discussed.

\section{Case report}

A 47-year-old woman came to the Department of Oral Surgery, Nihon University School of Dentistry at Matsudo, because of pain and swelling in the left buccal region in April, 1985. Her past medical history showed she had undergone surgery for appendicitis in 1961, nd her upper left first molar had been extracted at a dental clinic in 1963. At that time, the root-tip of the molar had remained in the left sinus cavity. She had sub equently complained several times of purulent nasal secretion on the left side and spontaneous pain in the left buccal region. In 1983 she visited an otolaryngology clinic because the symptoms of secretion and pain had become severe. She was diagnosed as having acute maxillary sinusitis and was treated with antibiotics for several days, after which the symptoms im-

川名以徳, 山本浩嗣*, 泉 廣次：Departments of Oral Surgery and Pathology*, Nihon University School of Dentistry at Matsudo, Matsudo, Chiba 271, Japan. 
proved. However, she suffered from the same symptoms again in February 1985. At the time of her first visit to our hospital, a diffuse swelling with spontaneous pain in the left buccal region was found. Slight swelling of the left mandibular lymphnodes was noticed, but no nasal secretion or fever was seen. The intraoral picture was as follows:

\begin{tabular}{|c|c|c|c|c|c|c|c|c|c|c|}
\hline Brid, & & & & & & & & & & Bridge \\
\hline 7 & 5 & 4 & 3 & 2 & 1 & 1 & 2 & 3 & 4 & 5 \\
\hline 76 & 5 & 4 & 3 & 2 & 1 & 1 & 2 & 3 & 4 & 6 \\
\hline
\end{tabular}

Compression pain and swelling were recognized at the upper left buccal gingiva of the first and second premolar root areas. The mucosal color in the same areas was reddish. By needle puncture, approx. $5 \mathrm{ml}$ of purulent, yellow, liquid-like pus was aspirated from the left maxillary sinus, after which the pain disappeared. Waters' radiographic examination showed massive opacification of the left maxillary sinus. In a panoramic radiograph, the root-tip of the upper first molar was identified in the center of the left maxillary sinus (Fig. 1). She was diagnosed as having odontogenic maxillary sinusitis. In May 1985, Caldwell-Luc procedure was carried out under intravenous sedation. Purulent yellow-grayish pus and green rubbery material surrounding the root at the adjacent orbit were found in the left maxillary sinus. The mucosal lesion was removed, and the underlying bone was found to be intact. After the operation, the symptoms associated with the left maxillary sinus improved.

\section{Pathological Findings}

Macroscopically, the removed materials consisted of edematous sinus mucosae with partially necrotic and hemorrhagic areas. The root-tip of the upper first molar was also recognized to have surrounding grayish rubbery material (Fig. 2). Microscopically, the histological examination showed moderate to severe inflammatory cell infiltration, fibrosis, edema and capillary dilatation in the submucosa covered with rough cuboidal and ciliated columnar epithelia. Glandular dilatation

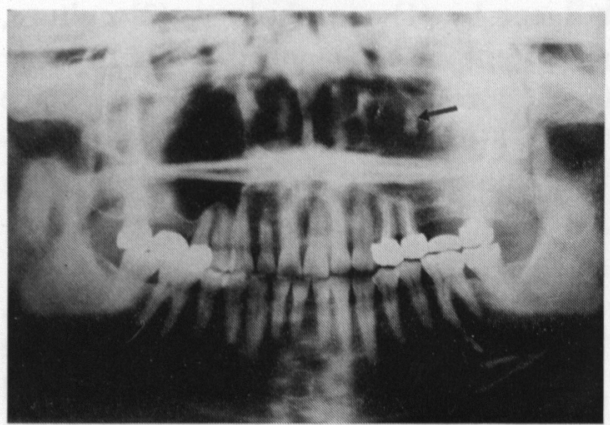

Fig. 1 Panoramic radiograph showing a mass opacification and a root-tip of the upper first molar (arrow) in the left maxillary sinus

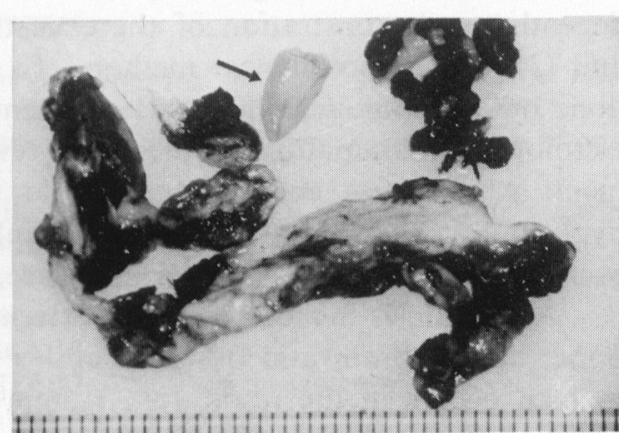

Fig. 2 Macroscopical finding of sinus revealing thick mucosae with partially necrotic areas and a root-tip of upper first molar (arrow) 


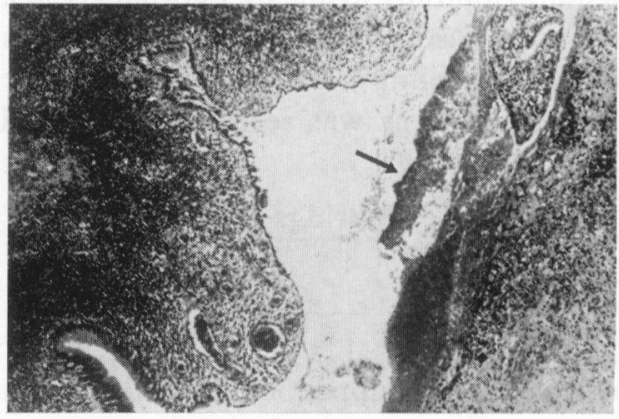

Fig. 3 Microscopic view of the mucosa showing respiratory epithelium with several infiltrating round cells, mainly plasma cells and lymphocytes and fungus coat in the cavity (arrow) (H.E. $\times 40$ )

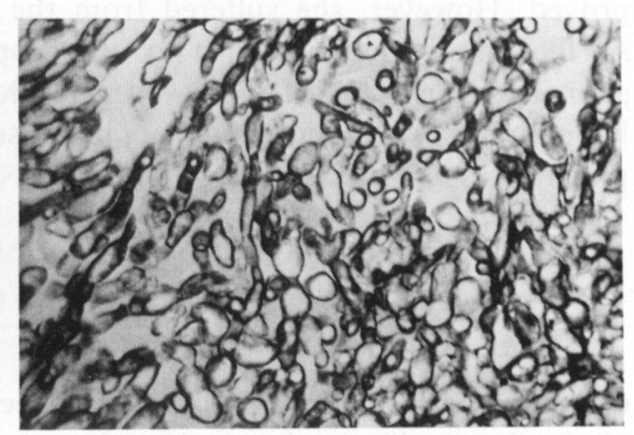

Fig. 4 High-power view of fungus coat showing a mass of fungal hyphae with the characteristic appearance of Aspergillus $($ GMS $\times 400)$

with mucoid retention was seen. A yellow whitish fungus coat, a so-called fungus ball, was found on the surface of the necrotic membrane (Fig. 3). The inflamed antral lining paste was shown to be composed of a mass of fungal hyphae with the characteristic appearance of Aspergillus. It was a dimorphic fungus of the ascomycete class. Its filaments were approximately 4 to $5 \mu \mathrm{m}$ in diameter and branched dichotomously at an angle of about $45^{\circ}$. The branching had a Y-shaped pattern with a characteristic unidirectional orientation by GMS staining (Fig. 4) and septa were also observed.

\section{Discussion}

The etiologic agents of aspergillosis mainly include a number of the Aspergillus fumigatus group, although more than 130 species of Aspergillus are recognized. In addition, several species belonging to other Aspergillus groups, particularly $A$. flavus, $A$. niger and $A$. terreus are also capable of causing aspergillosis, and are found worldwide ${ }^{[1]}$. In general, successful laboratory diagnosis of these organisms depends on demonstration of the causative agent by (1) morphology, (2) culture, and (3) indirect serological methods. In the present case, diagnosis was primarily done by morphology, which was sufficient to allow recognition of the disease, since histological examination of the sinus revealed the existence of typical fungal elements which were composed of septa and dichotomously branched Aspergillus hyphae. For species subclassification, culture methods would be necessary. However, the culture failed in the present case.

Aspergillosis invades the skin, external ear, orbit, sinus, bronchi, pleura, heart, bone, uterus, brain and other organs ${ }^{[2-4]}$. The most common form of the disease in man occurs in the respiratory system ${ }^{[1]}$. In Japan, over 160 cases of paranasal fungus infection had been reported in the literature up to $1982^{[5]}$. In addition, upon review up to 1986 , ca. 250 cases of fungal infection of the paranasal sinus were documented in the Japanese literature ${ }^{[5-8]}$. About half of these cases were aspergillosis, which was the most frequent in all paranasal fungus infections. 
Generally, Aspergillus is believed to be an opportunistic pathogen and is more commonly found in chronically ill and debilitated patients. In recent years, the widespread use of antibiotics, steroids as well as anticancer agents, has resulted in an increase of cases of fungus infection ${ }^{[9]}$. Furthermore, Veress et al. ${ }^{[10]}$ believe that two additional factors, i.e., a suitable anaerobic environment and reduced immunologic response, may play an important role in paranasal sinus aspergillosis.

Aspergillosis of the paranasal sinus due to odontogenic infection is rare. In Japan, only 6 among all cases were caused by odontogenic sinusitis due to Aspergillus infection. The present case was followed by odontogenic sinusitis originating from the upper left first molar root, and when local infective resistance was reduced, the opportunistic infection was considered to have occurred.

Three clinical types of aspergillosis in the paranasal sinuses have been reported; the non-invasive type, the invasive type and the fulminant type ${ }^{[11,12]}$. The noninvasive type resembles bacterial sinusitis, while the invasive type may be present with extension into the cheek or orbit. The fulminant form of paranasal sinus aspergillosis occurs in immunodeficient patients who are mainly children with acute leukemia undergoing radiation therapy and chemotherapy ${ }^{[12]}$. In the non-invasive type, radiographs may show only clouding or opacification of the sinus. The invasive type is manifested radiologically as a paranasal sinus mass with orbital displacement and bony erosion. Bony destruction in radiographs was only recognized in about $1 / 5$ of cases in the Japanese literature. Thus, most cases of paranasal fungus infection were of the non-invasive type. Odontogenic aspergillosis of the paranasal sinus has always been of the non-invasive type in Japan. Histological examination shows a 'fungus ball' usually occupies a portion of the sinus cavity and inflammation is limited to the cavity in the non-invasive type. On the other hand, in the invasive type, inflammation is widespread and associated with bony destruction ${ }^{[1]}$. As for treatment of the non-invasive type, local surgery (Caldwell-Luc procedure) and aeration are useful ${ }^{[5,13-15]}$. Combination therapy involving surgery and chemotherapy using amphotericin $B$ is effective in the invasive type ${ }^{[16]}$. In the present case, the patient was cured by local surgery only.

\section{References}

[1] Chandler, F.W., Kaplan, W.K. and Ajello, L.: A colour atlas and textbook of the histopathology of mycotic diseases, Wolfe Medical Publications Ltd., London, 34-37, 1980

[2] Anderson, B.S., LunA, M.A. and MacCredie, K.B.: Systemic aspergillosis as cause of myocardial infection, Cancer, 9, 2146-2150, 1986

[3] Inuyama, Y., Ozu, R., Nakajima, K. and Katahara, S.: Clinical observation of fungus infection of paranasal sinuses, Practica Otologica, Kyoto, 69, 4, 325-335, 1976 (in Japanese)

[4] Kahn, F.W., Jone, J.M. and England, D.M.: The role of bronchoalveolar lavage in the diagnosis of invasive pulmonary aspergillosis, Am. J. Clin. Pathol., 86, 4, 518-523, 1986

[5] Suenaga, T., Sasaki, N., Fukuda, T., Tanaka, M. and Tamiya, Y.: Fungal infection of nasal and paranasal cavity, J. Otolaryngol. Jpn., 87, 1082-1088, 1984 (in Japanese)

[6] Enomoto, K., Hotate, T., Azuma, E., Asakura, K. and Kataura, A.: Exploratory maxillary sinusotomy in 100 cases, Oto-Rhino-Laryngology, 27, 5, 493-497, 1984 (in Japanese)

[7] Harada, H., Inoue, T., Hiraide, F., Hosokawa, S., Tanaka, E., Nishizawa, S. and Ogura, M.: Fungus infection of the nose, Oto-Rhino-Laryngology, Tokyo, 27, 3, 271-276, 1984 (in Japanese) 
[8] Masaki, M., Uchida, Y., Ohto, T. and Sugita, T.: Aspergillosis of the paranasal sinus, OtoRhino-Laryngology, Tokyo, 25, 2, 138-143, 1982 (in Japanese)

[9] Zimmerman, L.E.: Fatal fungal infections complicating other disease, Am. J. Clin., 25, 4665,1955

[10] Veress, B., Malik, O.A. and Teyeb, A.A.: Further observations on the primary paranasal aspergillus granuloma in Sudan: a morphological study of 46 cases, Am. J. Trop. Med. Hyg., 22, 6, 765-772, 1973

[11] HORA, J.F.: Primary aspergillosis of the paranasal sinuses and associated areas, Laryngoscope, 75, 768-773, 1965

[12] MacGill, T.J., Simpson, G. and Healy, G.B.: Fulminant aspergillosis of the nose and paranasal sinuses; a new clinical entity, Laryngoscope, 90, 748-754, 1980

[13] McGuirt, W.F. and Harrill, J.A.: Paranasal sinus aspergillosis, Laryngoscope, 89, 15631568,1979

[14] Miglet, A.W., Saunders, W.H. and Ayers, L.: Aspergillosis of the sphenoid sinus, Arch Otolaryngol., 104, 47-50, 1987

[15] Warder, F.R., Chikes, P.G. and Husdon, W.R.: Aspergillosis of paranasal sinus, Arch. Otolaryngol., 101, 683-685, 1972

[16] Bahdur, S., Kacker, S.K., D'Souza, B. and Chopra, P.: Paranasal sinus aspergillosis, J. Laryngol. Otol., 97, 863-867, 1983 